\title{
Efficient Dialogue State Tracking by Selectively Overwriting Memory
}

\author{
Sungdong Kim Sohee Yang Gyuwan Kim Sang-Woo Lee \\ Clova AI, NAVER Corp. \\ \{sungdong.kim, sh.yang, gyuwan.kim, sang.woo.lee\}@navercorp.com
}

\begin{abstract}
Recent works in dialogue state tracking (DST) focus on an open vocabulary-based setting to resolve scalability and generalization issues of the predefined ontology-based approaches. However, they are inefficient in that they predict the dialogue state at every turn from scratch. Here, we consider dialogue state as an explicit fixed-sized memory and propose a selectively overwriting mechanism for more efficient DST. This mechanism consists of two steps: (1) predicting state operation on each of the memory slots, and (2) overwriting the memory with new values, of which only a few are generated according to the predicted state operations. Our method decomposes DST into two sub-tasks and guides the decoder to focus only on one of the tasks, thus reducing the burden of the decoder. This enhances the effectiveness of training and DST performance. Our SOM-DST (Selectively Overwriting Memory for Dialogue State Tracking) model achieves state-of-theart joint goal accuracy with $51.72 \%$ in MultiWOZ 2.0 and $53.01 \%$ in MultiWOZ 2.1 in an open vocabulary-based DST setting. In addition, we analyze the accuracy gaps between the current and the ground truth-given situations and suggest that it is a promising direction to improve state operation prediction to boost the DST performance. ${ }^{1}$
\end{abstract}

\section{Introduction}

Building robust task-oriented dialogue systems has gained increasing popularity in both the research and industry communities (Chen et al., 2017). Dialogue state tracking (DST), one of the essential tasks in task-oriented dialogue systems (Zhong et al., 2018), is keeping track of user goals or intentions throughout a dialogue in the form of a set of slot-value pairs, i.e., dialogue state. Because the

\footnotetext{
${ }^{1}$ The code is available at github.com/clovaai/som-dst.
}

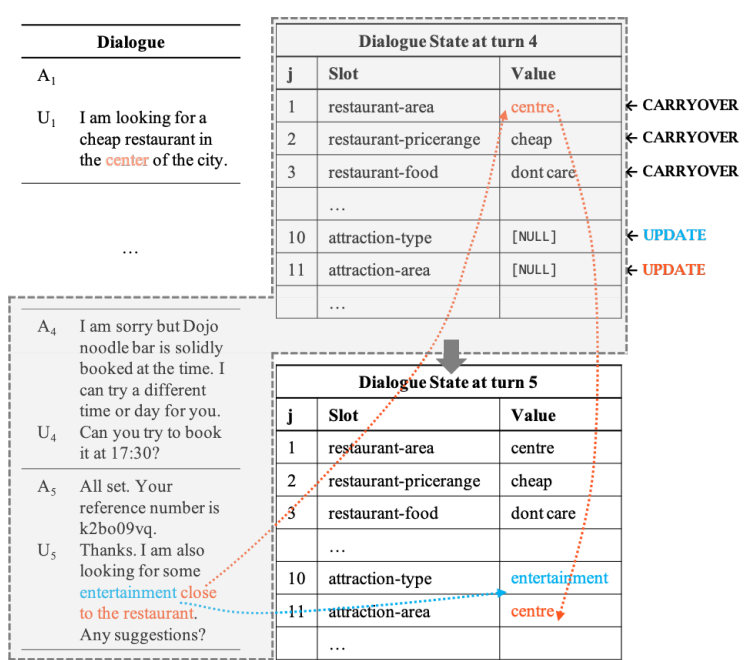

Figure 1: An example of how SOM-DST performs dialogue state tracking at a specific dialogue turn (in this case, fifth). The shaded part is the input to the model, and "Dialogue State at turn 5" at the right-bottom part is the output of the model. Here, UPDATE operation needs to be performed on the 10th and 11th slot. DST at this turn is challenging since the model requires reasoning over the long-past conversation. However, SOMDST can still robustly perform DST because the previous dialogue state is directly utilized like a memory.

next dialogue system action is selected based on the current dialogue state, an accurate prediction of the dialogue state has significant importance.

Traditional neural DST approaches assume that all candidate slot-value pairs are given in advance, i.e., they perform predefined ontology-based DST (Mrkšić et al., 2017; Zhong et al., 2018; Nouri and Hosseini-Asl, 2018; Lee et al., 2019). Most previous works that take this approach perform DST by scoring all possible slot-value pairs in the ontology and selecting the value with the highest score as the predicted value of a slot. Such an approach has been widely applied to datasets like DSTC2 and WOZ2.0, which have a relatively small ontology size. (Henderson et al., 2014; Wen et al., 2017) 
Although this approach simplifies the task, it has inherent limitations: (1) it is often difficult to obtain the ontology in advance, especially in a real scenario (Xu and $\mathrm{Hu}, 2018$ ), (2) predefined ontologybased DST cannot handle previously unseen slot values, and (3) the approach does not scale large since it has to go over all slot-value candidates at every turn to predict the current dialogue state. Indeed, recent DST datasets often have a large size of ontology; e.g., the total number of slot-value candidates in MultiWOZ 2.1 is 4510, while the numbers are much smaller in DSTC2 and WOZ2.0 as 212 and 99, respectively (Budzianowski et al., 2018).

To address these issues, recent methods employ an approach that either directly generates or extracts a value from the dialogue context for every slot, allowing open vocabulary-based DST (Lei et al., 2018; Gao et al., 2019; Wu et al., 2019; Ren et al., 2019). While this formulation is relatively more scalable and robust to handling unseen slot values, many of the previous works do not efficiently perform DST since they predict the dialogue state from scratch at every dialogue turn.

In this work, we focus on an open vocabularybased setting and propose SOM-DST (Selectively Overwriting Memory for Dialogue State Tracking). Regarding dialogue state as a memory that can be selectively overwritten (Figure 1), SOM-DST decomposes DST into two sub-tasks: (1) state operation prediction, which decides the types of the operations to be performed on each of the memory slots, and (2) slot value generation, which generates the values to be newly written on a subset of the memory slots (Figure 2). This decomposition allows our model to efficiently generate the values of only a minimal subset of the slots, while many of the previous works generate or extract the values of all slots at every dialogue turn. Moreover, this decomposition reduces the difficulty of DST in an open-vocabulary based setting by clearly separating the roles of the encoder and the decoder. Our encoder, i.e., state operation predictor, can focus on selecting the slots to pass to the decoder so that the decoder, i.e., slot value generator, can focus only on generating the values of those selected slots. To the best of our knowledge, our work is the first to propose such a selectively overwritable memorylike perspective and a discrete two-step approach on DST.

Our proposed SOM-DST achieves state-of-theart joint goal accuracy in an open vocabulary-based
DST setting on two of the most actively studied datasets: MultiWOZ 2.0 and MultiWOZ 2.1. Error analysis (Section 6.2) further reveals that improving state operation prediction can significantly boost the final DST accuracy.

In summary, the contributions of our work built on top of a perspective that considers dialogue state tracking as selectively overwriting memory are as follows:

- Enabling efficient DST, generating the values of a minimal subset of the slots by utilizing the previous dialogue state at each turn.

- Achieving state-of-the-art performance on MultiWOZ 2.0 and MultiWOZ 2.1 in an open vocabulary-based DST setting.

- Highlighting the potential of improving the state operating prediction accuracy in our proposed framework.

\section{Previous Open Vocabulary-based DST}

Many works on recent task-oriented dialogue datasets with a large scale ontology, such as MultiWOZ 2.0 and MultiWOZ 2.1, solve DST in an open vocabulary-based setting (Gao et al., 2019; Wu et al., 2019; Ren et al., 2019; Le et al., 2020a,b).

Wu et al. (2019) show the potential of applying the encoder-decoder framework (Cho et al., 2014a) to open vocabulary-based DST. However, their method is not computationally efficient because it performs autoregressive generation of the values for all slots at every dialogue turn.

Ren et al. (2019) tackle the drawback of the model of Wu et al. (2019), that their model generates the values of all slots at every dialogue turn, by using a hierarchical decoder. In addition, they come up with a new notion dubbed Inference Time Complexity (ITC) to compare the efficiency of different DST models. ITC is calculated using the number of slots $J$ and the number of corresponding slot values $M .^{2}$ Following their work, we also calculate ITC in Appendix B for comparison.

Le et al. (2020b) introduce another work that tackles the efficiency issue. To maximize the computational efficiency, they use a non-autoregressive decoder to generate the slot values of the current dialogue state at once. They encode the slot type information together with the dialogue context and

\footnotetext{
${ }^{2}$ The notations used in the work of Ren et al. (2019) are $n$ and $m$, respectively.
} 


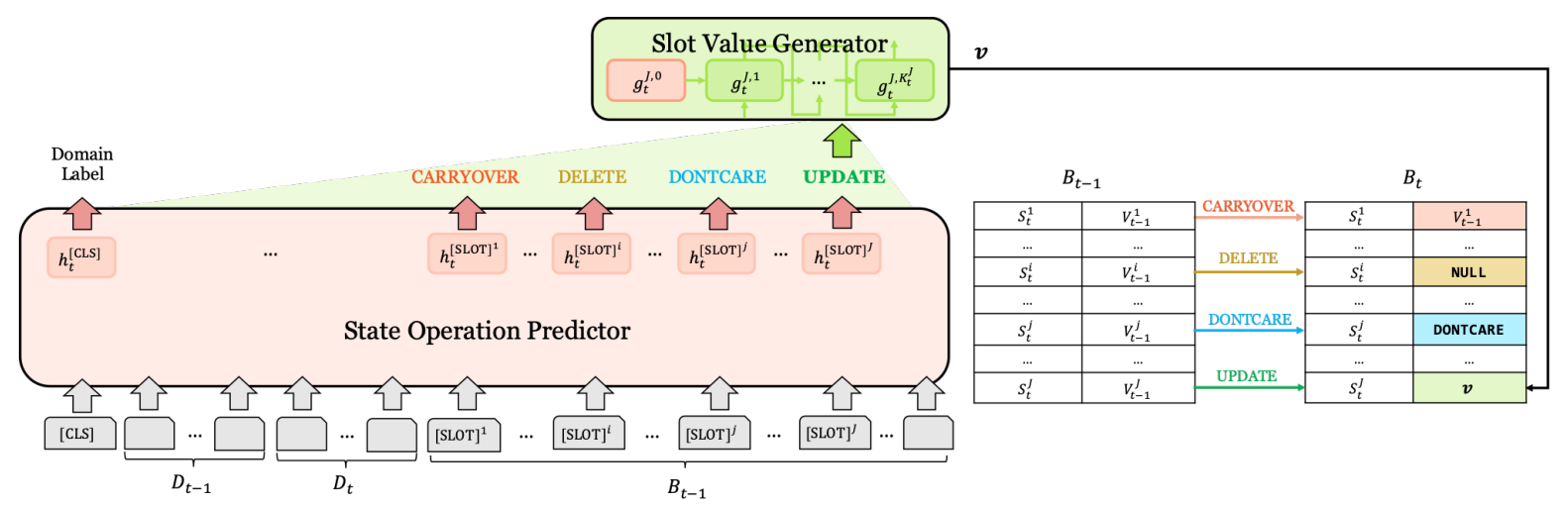

Figure 2: The overview of the proposed SOM-DST. SOM-DST takes the previous turn dialogue utterances $D_{t-1}$, current turn dialogue utterances $D_{t}$, and the previous dialogue state $B_{t-1}$ as the input and outputs the current dialogue state $B_{t}$. This is performed by two sub-components: state operation predictor and slot value generator. State operation predictor takes $D_{t-1}, D_{t}$, and $B_{t-1}$ as the input and predicts the operations to perform on each of the slots. Domain classification is jointly performed as an auxiliary task. Slot value generator generates the values for the slots that take UPDATE as the predicted operation. The value generation for a slot is done in an autoregressive manner.

the delexicalized dialogue context. They do not use the previous turn dialogue state as the input.

Le et al. (2020a) process the dialogue context in both domain-level and slot-level. They make the final representation to generate the values using a late fusion approach. They show that there is a performance gain when the model is jointly trained with response generation. However, they still generate the values of every slot at each turn, like Wu et al. (2019).

Gao et al. (2019) formulate DST as a reading comprehension task and propose a model named DST Reader that extracts the values of the slots from the input. They introduce and show the importance of the concept of a slot carryover module, i.e., a component that makes a binary decision whether to carry the value of a slot from the previous turn dialogue state over to the current turn dialogue state. The definition and use of discrete operations in our work is inspired by their work.

Zhang et al. (2019) target the issue of illformatted strings that generative models suffer from. In order to avoid this issue, they take a hybrid approach. For the slots they categorize as picklistbased slots, they use a predefined ontology-based approach as in the work of Lee et al. (2019); for the slots they categorize as span-based slots, they use a span extraction-based method like DST-Reader (Gao et al., 2019). However, their hybrid model shows lower performance than when they use only the picklist-based approach. Although their solely picklist-based model achieves state-of-the-art joint accuracy in MultiWOZ 2.1, it is done in a prede- fined ontology-based setting, and thus cannot avoid the scalability and generalization issues of predefined ontology-based DST.

\section{Selectively Overwriting Memory for Dialogue State Tracking}

Figure 2 illustrates the overview of SOM-DST. To describe the proposed SOM-DST, we formally define the problem setting in our work.

Dialogue State We define the dialogue state at turn $t, \mathcal{B}_{t}=\left\{\left(S^{j}, V_{t}^{j}\right) \mid 1 \leq j \leq J\right\}$, as a fixed-sized memory whose keys are slots $S^{j}$ and values are the corresponding slot value $V_{t}^{j}$, where $J$ is the total number of such slots. Following the convention of MultiWOZ 2.0 and MultiWOZ 2.1, we use the term "slot" to refer to the concatenation of a domain name and a slot name.

Special Value There are two special values NULL and DONTCARE. NULL means that no information is given about the slot up to the turn. For instance, the dialogue state before the beginning of any dialogue $\mathcal{B}_{0}$ has only NULL as the value of all slots. DONTCARE means that the slot neither needs to be tracked nor considered important in the dialogue at that time. ${ }^{3}$

Operation At every turn $t$, an operation $r_{t}^{j} \in \mathcal{O}=$ \{CARRYOVER, DELETE, DONTCARE, UPDATE\} is chosen by the state operation predictor (Section

\footnotetext{
${ }^{3}$ Such notions of "none value" and "dontcare value" appear in the previous works as well (Wu et al., 2019; Gao et al., 2019; Le et al., 2020b; Zhang et al., 2019).
} 
3.1) and performed on each slot $S^{j}$ to set its current turn corresponding value $V_{t}^{j}$. When an operation is performed, it either keeps the slot value unchanged (CARRYOVER) or changes it to some value different from the previous one (DELETE, DONTCARE, and UPDATE) as the following.

$$
V_{t}^{j}= \begin{cases}V_{t-1}^{j} & \text { if } r_{t}^{j}=\text { CARRYOVER } \\ \text { NULL } & \text { if } r_{t}^{j}=\text { DELETE } \\ \text { DONTCARE } & \text { if } r_{t}^{j}=\text { DONTCARE } \\ v & \text { if } r_{t}^{j}=\text { UPDATE }\end{cases}
$$

The operations that set the value of a slot to a special value (DELETE to NULL and DONTCARE to DONTCARE, respectively) are chosen only when the previous slot value $V_{t-1}^{j}$ is not the corresponding special value. UPDATE operation requires the generation of a new value $v \notin$ $\left\{V_{t-1}^{j}\right.$, NULL, DONTCARE $\}$ by slot value generator (Section 3.2).

State operation predictor performs state operation prediction as a classification task, and slot value generator performs slot value generation to find out the values of the slots on which UPDATE should be performed. The two components of SOM-DST are jointly trained to predict the current turn dialogue state.

\subsection{State Operation Predictor}

Input Representation We denote the representation of the dialogue utterances at turn $t$ as $D_{t}=$ $A_{t} \oplus ; \oplus U_{t} \oplus[\mathrm{SEP}]$, where $A_{t}$ is the system response and $U_{t}$ is the user utterance. ; is a special token used to mark the boundary between $A_{t}$ and $U_{t}$, and $[\mathrm{SEP}]$ is a special token used to mark the end of a dialogue turn. We denote the representation of the dialogue state at turn $t$ as $B_{t}=B_{t}^{1} \oplus \ldots \oplus B_{t}^{J}$, where $B_{t}^{j}=$ [SLOT $^{j} \oplus S^{j} \oplus-\oplus V_{t}^{j}$ is the representation of the $j$-th slot-value pair. - is a special token used to mark the boundary between a slot and a value. $\left[\mathrm{SLOT}^{j}\right]^{j}$ is a special token used to aggregate the information of the $j$-th slot-value pair into a single vector, like the use case of [CLS] token in BERT (Devlin et al., 2019). In this work, we use the same special token $[\mathrm{SLOT}]$ for all $[\mathrm{SLOT}]^{j}$. Our state operation predictor employs a pretrained BERT encoder. The input tokens to the state operation predictor are the concatenation of the previous turn dialog utterances, the current turn dialog utter- ances, and the previous turn dialog state: ${ }^{4}$

$$
X_{t}=[\mathrm{CLS}] \oplus D_{t-1} \oplus D_{t} \oplus B_{t-1},
$$

where [CLS] is a special token added in front of every turn input. Using the previous dialogue state as the input serves as an explicit, compact, and informative representation of the dialogue history for the model.

When the value of the $j$-th slot at time $t-1$, i.e., $V_{t-1}^{j}$, is NULL, we use a special token [NULL] as the input. When the value is DONTCARE, we use the string "dont care" to take advantage of the semantics of the phrase "don't care" that the pretrained BERT encoder would have already learned.

The input to BERT is the sum of the embeddings of the input tokens $X_{t}$, segment id embeddings, and position embeddings. For the segment id, we use 0 for the tokens that belong to $D_{t-1}$ and 1 for the tokens that belong to $D_{t}$ or $B_{t-1}$. The position embeddings follow the standard choice of BERT.

Encoder Output The output representation of the encoder is $H_{t} \in \mathbb{R}^{\left|X_{t}\right| \times d}$, and $h_{t}^{[\mathrm{CLS}]}, h_{t}^{\left[\mathrm{SLOT}^{j}\right.} \in$ $\mathbb{R}^{d}$ are the outputs that correspond to [CLS] and $[\mathrm{SLOT}]^{j}$, respectively. $h_{t}^{X}$, the aggregated sequence representation of the entire input $X_{t}$, is obtained by a feed-forward layer with a learnable parameter $W_{\text {pool }} \in \mathbb{R}^{d \times d}$ as:

$$
h_{t}^{X}=\tanh \left(W_{\text {pool }} h_{t}^{[\mathrm{CLS}]}\right) .
$$

State Operation Prediction State operation prediction is a four-way classification performed on top of the encoder output for each slot representation $h_{t}^{[\mathrm{SLOT}]^{j}}$ :

$$
P_{o p r, t}^{j}=\operatorname{softmax}\left(W_{o p r} h_{t}^{[\mathrm{SLOT}]^{j}}\right),
$$

where $W_{\text {opr }} \in \mathbb{R}^{|\mathcal{O}| \times d}$ is a learnable parameter and $P_{o p r, t}^{j} \in \mathbb{R}^{|\mathcal{O}|}$ is the probability distribution over operations for the $j$-th slot at turn $t$. In our formulation, $|\mathcal{O}|=4$, because $\mathcal{O}=\{$ CARRYOVER, DELETE, DONTCARE, UPDATE $\}$.

Then, the operation is determined by $r_{t}^{j}=$ $\operatorname{argmax}\left(P_{o p r, t}^{j}\right)$ and the slot value generation is performed on only the slots whose operation is UPDATE. We define the set of the slot indices which require the value generation as $\mathbb{U}_{t}=\left\{j \mid r_{t}^{j}=\right.$ UPDATE $\}$, and its size as $J_{t}^{\prime}=\left|\mathbb{U}_{t}\right|$.

\footnotetext{
${ }^{4}$ We use only the previous turn dialogue utterances $D_{t-1}$ as the dialogue history, i.e., the size of the dialogue history is 1 . This is because our model assumes Markov property in dialogues as a part of the input, the previous turn dialogue state $B_{t-1}$, can serve as a compact representation of the whole dialogue history.
} 


\subsection{Slot Value Generator}

For each $j$-th slot such that $j \in \mathbb{U}_{t}$, the slot value generator generates a value. Our slot value generator differs from the generators of many of the previous works because it generates the values for only $J_{t}^{\prime}$ number of slots, not $J$. In most cases, $J_{t}^{\prime} \ll J$, so this setup enables an efficient computation where only a small number of slot values are newly generated.

We use Gated Recurrent Unit (GRU) (Cho et al., 2014b) decoder like Wu et al. (2019). GRU is initialized with $g_{t}^{j, 0}=h_{t}^{\mathrm{X}}$ and $e_{t}^{j, 0}=h_{t}^{[\text {SLOT }]^{j}}$, and recurrently updates the hidden state $g_{t}^{j, k} \in \mathbb{R}^{d}$ by taking a word embedding $e_{t}^{j, k}$ as the input until [EOS] token is generated:

$$
g_{t}^{j, k}=\operatorname{GRU}\left(g_{t}^{j, k-1}, e_{t}^{j, k}\right) .
$$

The decoder hidden state is transformed to the probability distribution over the vocabulary at the $k$-th decoding step, where $E \in \mathbb{R}^{d_{v c b} \times d}$ is the word embedding matrix shared across the encoder and the decoder, such that $d_{v c b}$ is the vocabulary size.

$$
P_{v c b, t}^{j, k}=\operatorname{softmax}\left(E g_{t}^{j, k}\right) \in \mathbb{R}^{d_{v c b}} .
$$

As the work of Wu et al. (2019), we use the softgated copy mechanism (See et al., 2017) to get the final output distribution $P_{v a l, t}^{j, k}$ over the candidate value tokens:

$$
\begin{aligned}
& P_{c t x, t}^{j, k}=\operatorname{softmax}\left(H_{t} g_{t}^{j, k}\right) \in \mathbb{R}^{\left|X_{t}\right|}, \\
& P_{v a l, t}^{j, k}=\alpha P_{v c b, t}^{j, k}+(1-\alpha) P_{c t x, t}^{j, k},
\end{aligned}
$$

such that $\alpha$ is a scalar value computed as:

$$
\alpha=\operatorname{sigmoid}\left(W_{1}\left[g_{t}^{j, k} ; e_{t}^{j, k} ; c_{t}^{j, k}\right]\right),
$$

where $W_{1} \in \mathbb{R}^{1 \times(3 d)}$ is a learnable parameter and $c_{t}^{j, k}=P_{c t x, t}^{j, k} H_{t} \in \mathbb{R}^{d}$ is a context vector.

\subsection{Objective Function}

During training, we jointly optimize both state operation predictor and slot value generator.

State Operation Predictor In addition to the state operation classification, we use domain classification as an auxiliary task to force the model to learn the correlation of slot operations and domain transitions in between dialogue turns. Domain classification is done with a softmax layer on top of $h_{t}^{X}$ :

$$
P_{d o m, t}=\operatorname{softmax}\left(W_{d o m} h_{t}^{X}\right),
$$

where $W_{\text {dom }} \in \mathbb{R}^{d_{\text {dom }} \times d}$ is a learnable parameter and $P_{d o m, t} \in \mathbb{R}^{d_{d o m}}$ is the probability distribution over domains at turn $t . d_{d o m}$ is the number of domains defined in the dataset.

The loss for each of state operation classification and domain classification is the average of the negative log-likelihood, as follows:

$$
\begin{aligned}
& L_{o p r, t}=-\frac{1}{J} \sum_{j=1}^{J}\left(Y_{o p r, t}^{j}\right)^{\top} \log P_{o p r, t}^{j}, \\
& L_{d o m, t}=-\left(Y_{d o m, t}\right)^{\top} \log P_{d o m, t},
\end{aligned}
$$

where $Y_{\text {dom,t }} \in \mathbb{R}^{d_{d o m}}$ is the one-hot vector for the ground truth domain and $Y_{o p r, t}^{j} \in \mathbb{R}^{|\mathcal{O}|}$ is the one-hot vector for the ground truth operation for the $j$-th slot.

Slot Value Generator The objective function to train slot value generator is also the average of the negative log-likelihood:

$$
L_{s v g, t}=-\frac{1}{\left|\mathbb{U}_{t}\right|} \sum_{j \in \mathbb{U}_{t}} \frac{1}{K_{t}^{j}} \sum_{k=1}^{K_{t}^{j}}\left(Y_{v a l, t}^{j, k}\right)^{\top} \log P_{v a l, t}^{j, k},
$$

where $K_{t}^{j}$ is the number of tokens of the ground truth value that needs to be generated for the $j$-th slot. $Y_{v a l, t}^{j, k} \in \mathbb{R}^{d_{v c b}}$ is the one-hot vector for the ground truth token that needs to be generated for the $j$-th slot at the $k$-th decoding step.

Therefore, the final joint loss $L_{j o i n t, t}$ to be minimized at dialogue turn $t$ is the sum of the losses mentioned above:

$$
L_{j o i n t, t}=L_{o p r, t}+L_{d o m, t}+L_{s v g, t} .
$$

\section{Experimental Setup}

\subsection{Datasets}

We use MultiWOZ 2.0 (Budzianowski et al., 2018) and MultiWOZ 2.1 (Eric et al., 2019) as the datasets in our experiments. These datasets are two of the largest publicly available multi-domain taskoriented dialogue datasets, including about 10,000 dialogues within seven domains. MultiWOZ 2.1 is a refined version of MultiWOZ 2.0 in which the annotation errors are corrected. ${ }^{5}$

Following Wu et al. (2019), we use only five domains (restaurant, train, hotel, taxi, attraction)

\footnotetext{
2.1 .
} 
excluding hospital and police. ${ }^{6}$ Therefore, the number of domains $d_{d o m}$ is 5 and the number of slots $J$ is 30 in our experiments. We use the script provided by Wu et al. (2019) to preprocess the datasets. ${ }^{7}$

\subsection{Training}

We employ the pretrained BERT-base-uncased model $^{8}$ for state operation predictor and one GRU (Cho et al., 2014b) for slot value generator. The hidden size of the decoder is the same as that of the encoder, $d$, which is 768 . The token embedding matrix of slot value generator is shared with that of state operation predictor. We use BertAdam as our optimizer (Kingma and Ba, 2015). We use greedy decoding for slot value generator.

The encoder of state operation predictor makes use of a pretrained model, whereas the decoder of slot value generator needs to be trained from scratch. Therefore, we use different learning rate schemes for the encoder and the decoder. We set the peak learning rate and warmup proportion to $4 \mathrm{e}-5$ and 0.1 for the encoder and $1 \mathrm{e}-4$ and 0.1 for the decoder, respectively. We use a batch size of 32 and set the dropout (Srivastava et al., 2014) rate to 0.1 . We also utilize word dropout (Bowman et al., 2016) by randomly replacing the input tokens with the special [UNK] token with the probability of 0.1 . The max sequence length for all inputs is fixed to 256 .

We train state operation predictor and slot value generator jointly for 30 epochs and choose the model that reports the best performance on the validation set. During training, we use the ground truth state operations and the ground truth previous turn dialogue state instead of the predicted ones. When the dialogue state is fed to the model, we randomly shuffle the slot order with a rate of 0.5. This is to make state operation predictor exploit the semantics of the slot names and not rely on the position of the slot tokens or a specific slot order. During inference or when the slot order is not shuffled, the slots are sorted alphabetically. We use teacher forcing $50 \%$ of the time to train the decoder.

All experiments are performed on NAVER Smart Machine Learning (NSML) platform (Sung et al., 2017; Kim et al., 2018). All the reported results of SOM-DST are averages over ten runs.

\footnotetext{
${ }^{6}$ The excluded domains take up only a small portion of the dataset and do not even appear in the test set.

${ }^{7}$ github.com/jasonwu0731/trade-dst

${ }^{8}$ github.com/huggingface/transformers
}

\subsection{Baseline Models}

We compare the performance of SOM-DST with both predefined ontology-based models and open vocabulary-based models.

FJST uses a bidirectional LSTM to encode the dialogue history and uses a feed-forward network to predict the value of each slot (Eric et al., 2019).

HJST is proposed together with FJST; it encodes the dialogue history using an LSTM like FJST but uses a hierarchical network (Eric et al., 2019).

SUMBT exploits BERT-base as the encoder for the dialogue context and slot-value pairs. After encoding them, it scores every candidate slot-value pair in a non-parametric manner using a distance measure (Lee et al., 2019).

HyST employs a hierarchical RNN encoder and takes a hybrid approach that incorporates both a predefined ontology-based setting and an open vocabulary-based setting (Goel et al., 2019).

DST Reader formulates the problem of DST as an extractive QA task; it uses BERT-base to make the contextual word embeddings and extracts the value of the slots from the input as a span (Gao et al., 2019).

TRADE encodes the whole dialogue context with a bidirectional GRU and decodes the value for every slot using a copy-augmented GRU decoder (Wu et al., 2019).

COMER uses BERT-large as a feature extractor and a hierarchical LSTM decoder to generate the current turn dialogue state itself as the target sequence (Ren et al., 2019).

NADST uses a Transformer-based nonautoregressive decoder to generate the current turn dialogue state (Le et al., 2020b).

ML-BST uses a Transformer-based architecture to encode the dialogue context with the domain and slot information and combines the outputs in a late fusion approach. Then, it generates the slot values and the system response jointly (Le et al., 2020a).

DS-DST uses two BERT-base encoders and takes a hybrid approach of predefined ontology-based DST and open vocabulary-based DST. It defines picklist-based slots for classification similarly to SUMBT and span-based slots for span extraction like DST Reader (Zhang et al., 2019). 
Table 1: Joint goal accuracy on the test set of MultiWOZ 2.0 and 2.1. * indicates a result borrowed from Eric et al. (2019). HyST and DS-DST use a hybrid approach, partially taking advantage of the predefined ontology. $\dagger$ indicates the case where BERT-large is used for our model.

\begin{tabular}{lrr}
\hline & $\begin{array}{rr}\text { MultiWOZ } \\
2.0\end{array}$ & $\begin{array}{r}\text { MultiWOZ } \\
\text { Predefined Ontology }\end{array}$ \\
\hline HJST* $^{*}$ (Eric et al., 2019) & 38.40 & 35.55 \\
FJST* (Eric et al., 2019) & 40.20 & 38.00 \\
SUMBT (Lee et al., 2019) $^{*}$ & 42.40 & - \\
HyST* (Goel et al., 2019) & 42.33 & 38.10 \\
DS-DST (Zhang et al., 2019) & - & 51.21 \\
DST-picklist (Zhang et al., 2019) & - & $\mathbf{5 3 . 3 0}$ \\
\hline Open Vocabulary & & \\
\hline DST Reader* (Gao et al., 2019) & 39.41 & 36.40 \\
TRADE* (Wu et al., 2019) & 48.60 & 45.60 \\
COMER (Ren et al., 2019) & 48.79 & - \\
NADST (Le et al., 2020b) & 50.52 & 49.04 \\
ML-BST (Le et al., 2020a) & - & 50.91 \\
SOM-DST (ours) & $\mathbf{5 1 . 7 2}$ & $\mathbf{5 3 . 0 1}$ \\
\hline SOM-DST ${ }^{\dagger}$ (ours) & $\mathbf{5 2 . 3 2}$ & $\mathbf{5 3 . 6 8}$ \\
\hline
\end{tabular}

DST-picklist is proposed together with DS-DST and uses a similar architecture, but it performs only predefined ontology-based DST considering all slots as picklist-based slots (Zhang et al., 2019).

\section{Experimental Results}

\subsection{Joint Goal Accuracy}

Table 1 shows the joint goal accuracy of SOM-DST and other models on the test set of MultiWOZ 2.0 and MultiWOZ 2.1. Joint goal accuracy is an accuracy which checks whether all slot values predicted at a turn exactly match the ground truth values.

As shown in the table, SOM-DST achieves state-of-the-art performance in an open vocabularybased setting. Interestingly, on the contrary to the previous works, our model achieves higher performance on MultiWOZ 2.1 than on MultiWOZ 2.0. This is presumably because our model, which explicitly uses the dialogue state labels as input, benefits more from the error correction on the state annotations done in MultiWOZ 2.1. ${ }^{9}$

\footnotetext{
${ }^{9}$ Eric et al. (2019) report that the correction of the annotations done in MultiWOZ 2.1 changes about $32 \%$ of the state annotations of MultiWOZ 2.0, which indicates that MultiWOZ 2.0 consists of many annotation errors.
}

Table 2: Domain-specific results on the test set of MultiWOZ 2.1. Our model outperforms other models in taxi and train domains.

\begin{tabular}{llrr}
\hline Domain & Model & $\begin{array}{r}\text { Joint } \\
\text { Accuracy }\end{array}$ & $\begin{array}{r}\text { Slot } \\
\text { Accuracy }\end{array}$ \\
\hline \multirow{2}{*}{ Attraction } & NADST & 66.83 & 98.79 \\
& ML-BST & $\mathbf{7 0 . 7 8}$ & 99.06 \\
& SOM-DST (ours) & 69.83 & 98.86 \\
\hline \multirow{2}{*}{ Hotel } & NADST & 48.76 & 97.70 \\
& ML-BST & 49.52 & 97.50 \\
& SOM-DST (ours) & $\mathbf{4 9 . 5 3}$ & 97.35 \\
\hline \multirow{2}{*}{ Restaurant } & NADST & 65.37 & 98.78 \\
& ML-BST & $\mathbf{6 6 . 5 0}$ & 98.76 \\
& SOM-DST (ours) & 65.72 & 98.56 \\
\hline Taxi & NADST & 33.80 & 96.69 \\
& ML-BST & 23.05 & 96.42 \\
& SOM-DST (ours) & $\mathbf{5 9 . 9 6}$ & 98.01 \\
\hline Train & NADST & 62.36 & 98.36 \\
& ML-BST & 65.12 & 90.22 \\
& SOM-DST (ours) & $\mathbf{7 0 . 3 6}$ & 98.67 \\
\hline
\end{tabular}

\subsection{Domain-Specific Accuracy}

Table 2 shows the domain-specific results of our model and the concurrent works which report such results (Le et al., 2020a,b). Domain-specific accuracy is the accuracy measured on a subset of the predicted dialogue state, where the subset consists of the slots specific to a domain.

While the performance is similar to or a little lower than that of other models in other domains, SOM-DST outperforms other models in taxi and train domains. This implies that the state-of-the-art joint goal accuracy of our model on the test set comes mainly from these two domains.

A characteristic of the data from these domains is that they consist of challenging conversations; the slots of these domains are filled with more diverse values than other domains, ${ }^{10}$ and there are more than one domain changes, i.e., the user changes the conversation topic during a dialogue more than once. For a specific example, among the dialogues where the domain switches more than once, the number of conversations that end in taxi domain is ten times more than in other cases. A more detailed statistics are given in Table 10 in Appendix A.

Therefore, we assume our model performs relatively more robust DST in such challenging conversations. We conjecture that this strength attributes to the effective utilization of the previous turn dialogue state in its explicit form, like using a memory;

\footnotetext{
${ }^{10}$ The statistics of the slot value vocabulary size are shown in Table 9 in Appendix A.
} 
Table 3: Joint goal accuracy on the MultiWOZ 2.1 test set when the four-way state operation prediction changes to two-way, three-way, or six-way.

\begin{tabular}{llr}
\hline & \multicolumn{1}{c}{ State Operations } & $\begin{array}{r}\text { Joint } \\
\text { Accuracy }\end{array}$ \\
\hline 4 & CARRYOVER, DELETE, & 53.01 \\
& DONTCARE, UPDATE & \\
\hline 2 & CARRYOVER, NON-CARRYOVER & 52.06 \\
3 & CARRYOVER, DONTCARE, UPDATE & 52.63 \\
3 & CARRYOVER, DELETE, UPDATE & 52.64 \\
6 & CARRYOVER, DELETE, & 52.97 \\
& DONTCARE, UPDATE, YES, NO & \\
\hline
\end{tabular}

the model can explicitly keep even the information mentioned near the beginning of the conversation and directly copy the values from this memory whenever necessary. Figure 1 shows an example of a complicated conversation in MultiWOZ 2.1, where our model accurately predicts the dialogue state. More sample outputs of SOM-DST are provided in Appendix C.

\section{Analysis}

\subsection{Choice of State Operations}

Table 3 shows the joint goal accuracy where the four-way state operation prediction changes to twoway, three-way, or six-way.

The joint goal accuracy drops when we use twoway state operation prediction, which is a binary classification of whether to (1) carry over the previous slot value to the current turn or (2) generate a new value, like Gao et al. (2019). We assume the reason is that it is better to separately model operations DELETE, DONTCARE, and UPDATE that correspond to the latter class of the binary classification, since the values of DELETE and DONTCARE tend to appear implicitly while the values for UPDATE are often explicitly expressed in the dialogue.

We also investigate the performance when only three operations are used or two more state operations, YES and NO, are used. YES and NO represent the cases where yes or no should be filled as the slot value, respectively. The performance drops in all of the cases.

\subsection{Error Analysis}

Table 4 shows the joint goal accuracy of the combinations of the cases where the ground truth is used or not for each of the previous turn dialogue state, state operations at the current turn, and slot

Table 4: Joint goal accuracy of the current and the ground truth-given situations. Relative error rate is the proportion of the error when $100 \%$ is set as the error where no ground truth is used for SOP and SVG. (GT: Ground Truth, SOP: State Operation Prediction, SVG: Slot Value Generation, Pred: Predicted)

\begin{tabular}{cccrr}
\hline & GT & GT & $\begin{array}{r}\text { Joint } \\
\text { Accuracy }\end{array}$ & $\begin{array}{r}\text { Relative } \\
\text { Error Rate }\end{array}$ \\
\hline & & & 53.01 & 100.0 \\
Pred $B_{t-1}$ & & $\checkmark$ & 56.37 & 92.85 \\
(w/ Error & $\checkmark$ & & 89.85 & 21.60 \\
Propagation) & $\checkmark$ & $\checkmark$ & 100.0 & 0.00 \\
\hline \multirow{2}{*}{ GT $B_{t-1}$} & & & 81.00 & 100.0 \\
(w/o Error & & $\checkmark$ & 82.80 & 90.53 \\
Propagation) & $\checkmark$ & & 96.27 & 19.63 \\
& $\checkmark$ & $\checkmark$ & 100.0 & 0.00 \\
\hline
\end{tabular}

values for UPDATE at the current turn. From this result, we analyze which of state operation predictor and slot value generator is more responsible for the error in the joint goal prediction, under the cases where error propagation occurs or not.

Among the absolute error of $46.99 \%$ made under the situation that error propagation occurs, i.e., the dialogue state predicted at the previous turn is fed to the model, it could be argued that $92.85 \%$ comes from state operation predictor, $21.6 \%$ comes from slot value generator, and $14.45 \%$ comes from both of the components. This indicates that at least $78.4 \%$ to $92.85 \%$ of the error comes from state operation predictor, and at least $7.15 \%$ to $21.6 \%$ of the error comes from slot value generator. ${ }^{11}$

Among the absolute error of $19 \%$ made under the error propagation-free situation, i.e., ground truth previous turn dialogue state is fed to the model, it could be argued that $90.53 \%$ comes from state operation predictor, $19.63 \%$ comes from slot value generator, and $10.16 \%$ comes from both of the components. This indicates that at least $80.37 \%$ to $90.53 \%$ of the error comes from state operation predictor, and at least $9.47 \%$ to $19.63 \%$ of the error comes from slot value generator.

Error propagation that comes from using the dialogue state predicted at the previous turn increases the error $2.47\left(=\frac{100-53.01}{100-81.00}\right)$ times. Both with and without error propagation, a relatively large amount

\footnotetext{
${ }^{11}$ The calculation of the numbers in the paragraph is done as follows. (The figures in the paragraph immediately below are calculated in the same way.)

$100-53.01=46.99$

$(100-56.37) / 46.99=92.85$

$(100-89.85) / 46.99=21.6$
} 
Table 5: Statistics of the number of state operations and the corresponding F1 scores of our model in MultiWOZ 2.1.

\begin{tabular}{lrrrr}
\hline & \multicolumn{3}{c}{ \# Operations } & F1 score \\
\cline { 2 - 4 } Operation Type & Train & Valid & Test & Test \\
\hline CARRYOVER & $1,584,757$ & 212,608 & 212,297 & 98.66 \\
UPDATE & 61,628 & 8,287 & 8,399 & 80.10 \\
DONTCARE & 1,911 & 155 & 235 & 32.51 \\
DELETE & 1,224 & 80 & 109 & 2.86 \\
\hline
\end{tabular}

Table 6: The minimum, average, and maximum number of slots whose values are generated at a turn, calculated on the test set of MultiWOZ 2.1.

\begin{tabular}{lrrr}
\hline Model & Min \# & Avg \# & Max \# \\
\hline TRADE & 30 & 30 & 30 \\
ML-BST & 30 & 30 & 30 \\
COMER & 0 & 5.72 & 18 \\
SOM-DST (ours) & 0 & 1.14 & 9 \\
\hline
\end{tabular}

Table 7: Average inference time per dialogue turn of MultiWOZ 2.1 test set, measured on Tesla V100 with a batch size of 1 . $\dagger$ indicates the case where BERT-large is used for our model.

\begin{tabular}{lrr}
\hline Model & Joint Accuracy & Latency \\
\hline TRADE & 45.60 & $340 \mathrm{~ms}$ \\
NADST & 49.04 & $26 \mathrm{~ms}$ \\
SOM-DST (ours) $_{\text {SOM-DST }^{\dagger} \text { (ours) }}$ & $\mathbf{5 3 . 0 1}$ & $27 \mathrm{~ms}$ \\
\hline
\end{tabular}

of error comes from state operation predictor, implying that a large room for improvement currently exists in this component. Improving the state operation prediction accuracy, e.g., by tackling the class imbalance shown in Table 5, may have the potential to increase the overall DST performance by a large margin.

\subsection{Efficiency Analysis}

In Table 6, we compare the number of slot values generated at a turn among various open vocabularybased DST models that use an autoregressive decoder.

The maximum number of slots whose values are generated by our model at a turn, i.e., the number of slots on which UPDATE should be performed, is 9 at maximum and only 1.14 on average in the test set of MultiWOZ 2.1.

On the other hand, TRADE and ML-BST generate the values of all the 30 slots at every turn of a dialogue. COMER generates only a subset of the slot values like our model, but it generates the val- ues of all the slots that have a non-NULL value at a turn, which is 18 at maximum and 5.72 on average.

Table 7 shows the latency of SOM-DST and several other models. We measure the inference time for a dialogue turn of MultiWOZ 2.1 on Tesla V100 with a batch size of 1 . The models used for comparison are those with official public implementations.

It is notable that the inference time of SOMDST is about 12.5 times faster than TRADE, which consists of only two GRUs. Moreover, the latency of SOM-DST is compatible with that of NADST, which explicitly uses non-autoregressive decoding, while SOM-DST achieves much higher joint goal accuracy. This shows the efficiency of the proposed selectively overwriting mechanism of SOM-DST, which generates only the minimal slot values at a turn.

In Appendix B, we also investigate Inference Time Complexity (ITC) proposed in the work of Ren et al. (2019), which defines the efficiency of a DST model using $J$, the number of slots, and $M$, the number of values of a slot.

\section{Conclusion}

We propose SOM-DST, an open vocabulary-based dialogue state tracker that regards dialogue state as an explicit memory that can be selectively overwritten. SOM-DST decomposes dialogue state tracking into state operation prediction and slot value generation. This setup makes the generation process efficient because the values of only a minimal subset of the slots are generated at each dialogue turn. SOM-DST achieves state-of-the-art joint goal accuracy on both MultiWOZ 2.0 and MultiWOZ 2.1 datasets in an open vocabulary-based setting. SOMDST effectively makes use of the explicit dialogue state and discrete operations to perform relatively robust DST even in complicated conversations. Further analysis shows that improving state operation prediction has the potential to increase the overall DST performance dramatically. From this result, we propose that tackling DST with our proposed problem definition is a promising future research direction.

\section{Acknowledgments}

The authors would like to thank the members of Clova AI for proofreading this manuscript. 


\section{References}

Samuel R. Bowman, Luke Vilnis, Oriol Vinyals, Andrew Dai, Rafal Jozefowicz, and Samy Bengio. 2016. Generating sentences from a continuous space. In Proceedings of The 20th SIGNLL Conference on Computational Natural Language Learning, pages 10-21, Berlin, Germany. Association for Computational Linguistics.

Paweł Budzianowski, Tsung-Hsien Wen, Bo-Hsiang Tseng, Inigo Casanueva, Stefan Ultes, Osman Ramadan, and Milica Gasic. 2018. Multiwoz - a largescale multi-domain wizard-of-oz dataset for taskoriented dialogue modelling. In EMNLP.

Hongshen Chen, Xiaorui Liu, Dawei Yin, and Jiliang Tang. 2017. A survey on dialogue systems: Recent advances and new frontiers. ACM SIGKDD Explorations Newsletter, 19(2):25-35.

Kyunghyun Cho, Bart van Merrienboer, Caglar Gulcehre, Dzmitry Bahdanau, Fethi Bougares, Holger Schwenk, and Yoshua Bengio. 2014a. Learning phrase representations using rnn encoder-decoder for statistical machine translation. In $E M N L P$.

Kyunghyun Cho, Bart Van Merriënboer, Dzmitry Bahdanau, and Yoshua Bengio. 2014b. On the properties of neural machine translation: Encoder-decoder approaches. In Proceedings of SSST-8, Eighth Workshop on Syntax, Semantics and Structure in Statistical Translation.

Jacob Devlin, Ming-Wei Chang, Kenton Lee, and Kristina Toutanova. 2019. Bert: Pre-training of deep bidirectional transformers for language understanding. In NAACL-HLT.

Mihail Eric, Rahul Goel, Shachi Paul, Abhishek Sethi, Sanchit Agarwal, Shuyag Gao, and Dilek HakkaniTur. 2019. Multiwoz 2.1: Multi-domain dialogue state corrections and state tracking baselines. arXiv preprint arXiv:1907.01669.

Shuyang Gao, Abhishek Sethi, Sanchit Agarwal, Tagyoung Chung, and Dilek Hakkani-Tur. 2019. Dialog state tracking: A neural reading comprehension approach. In SIGDIAL.

Rahul Goel, Shachi Paul, and Dilek Hakkani-Tür. 2019. Hyst: A hybrid approach for flexible and accurate dialogue state tracking. In Interspeech.

Matthew Henderson, Blaise Thomson, and Jason D Williams. 2014. The second dialog state tracking challenge. In SIGDIAL.

Hanjoo Kim, Minkyu Kim, Dongjoo Seo, Jinwoong Kim, Heungseok Park, Soeun Park, Hyunwoo Jo, KyungHyun Kim, Youngil Yang, Youngkwan Kim, et al. 2018. Nsml: Meet the mlaas platform with a real-world case study. arXiv preprint arXiv:1810.09957.

Diederik P Kingma and Jimmy Ba. 2015. Adam: A method for stochastic optimization. In ICLR.
Hung Le, Doyen Sahoo, Chenghao Liu, Nancy F. Chen, and Steven C.H. Hoi. 2020a. End-to-end multidomain task-oriented dialogue systems with multilevel neural belief tracker. In Submitted to ICLR 2020 .

Hung Le, Richard Socher, and Steven C.H. Hoi. 2020 b. Non-autoregressive dialog state tracking. In ICLR.

Hwaran Lee, Jinsik Lee, and Tae-Yoon Kim. 2019. Sumbt: Slot-utterance matching for universal and scalable belief tracking. In $A C L$.

Wenqiang Lei, Xisen Jin, Min-Yen Kan, Zhaochun Ren, Xiangnan He, and Dawei Yin. 2018. Sequicity: Simplifying task-oriented dialogue systems with single sequence-to-sequence architectures. In Proceedings of the 56th Annual Meeting of the Association for Computational Linguistics (Volume 1: Long Papers), pages 1437-1447, Melbourne, Australia. Association for Computational Linguistics.

Nikola Mrkšić, Diarmuid Ó Séaghdha, Tsung-Hsien Wen, Blaise Thomson, and Steve Young. 2017. Neural belief tracker: Data-driven dialogue state tracking. In $A C L$.

Elnaz Nouri and Ehsan Hosseini-Asl. 2018. Toward scalable neural dialogue state tracking model. In 2nd Conversational AI workshop on NeurIPS 2018.

Liliang Ren, Jianmo Ni, and Julian McAuley. 2019. Scalable and accurate dialogue state tracking via hierarchical sequence generation. In EMNLPIJCNLP.

Abigail See, Peter J Liu, and Christopher D Manning. 2017. Get to the point: Summarization with pointergenerator networks. In $A C L$.

Nitish Srivastava, Geoffrey Hinton, Alex Krizhevsky, Ilya Sutskever, and Ruslan Salakhutdinov. 2014. Dropout: a simple way to prevent neural networks from overfitting. JMLR, 15(1):1929-1958.

Nako Sung, Minkyu Kim, Hyunwoo Jo, Youngil Yang, Jingwoong Kim, Leonard Lausen, Youngkwan Kim, Gayoung Lee, Donghyun Kwak, Jung-Woo Ha, et al. 2017. Nsml: A machine learning platform that enables you to focus on your models. arXiv preprint arXiv:1712.05902.

Tsung-Hsien Wen, David Vandyke, Nikola Mrkšić, Milica Gasic, Lina M Rojas Barahona, Pei-Hao Su, Stefan Ultes, and Steve Young. 2017. A networkbased end-to-end trainable task-oriented dialogue system. In $E A C L$.

Chien-Sheng Wu, Andrea Madotto, Ehsan HosseiniAsl, Caiming Xiong, Richard Socher, and Pascale Fung. 2019. Transferable multi-domain state generator for task-oriented dialogue systems. In $A C L$.

Puyang Xu and Qi Hu. 2018. An end-to-end approach for handling unknown slot values in dialogue state tracking. In $A C L$. 
Jian-Guo Zhang, Kazuma Hashimoto, Chien-Sheng Wu, Philip S. Yu, Richard Socher, and Caiming Xiong. 2019. Find or classify? dual strategy for slotvalue predictions on multi-domain dialog state tracking. arXiv preprint arXiv:1910.03544.

Victor Zhong, Caiming Xiong, and Richard Socher. 2018. Global-locally self-attentive encoder for dialogue state tracking. In $A C L$. 


\section{A Data Statistics}

Table 8: Data Statistics of MultiWOZ 2.1.

\begin{tabular}{llrrrrrr}
\hline \multirow{2}{*}{ Domain } & \multirow{2}{*}{ Slots } & \multicolumn{2}{c}{ \# of Dialogues } & \multicolumn{3}{c}{ \# of Turns } \\
\cline { 2 - 7 } Attraction & area, name, type & Train & Valid & Test & Train & Valid & Test \\
\hline Hotel & $\begin{array}{l}\text { price range, type, parking, book stay, book day, } \\
\text { book people, area, stars, internet, name }\end{array}$ & 2,717 & 401 & 395 & 8,073 & 1,220 & 1,256 \\
\hline Restaurant & $\begin{array}{l}\text { food, price range, area, name, book time, book } \\
\text { day, book people }\end{array}$ & 3,813 & 416 & 394 & 14,793 & 1,781 & 1,756 \\
& leave at, destination, departure, arrive by & 1,654 & 207 & 195 & 4,618 & 690 & 654 \\
\hline Taxi & $\begin{array}{l}\text { destination, day, departure, arrive by, book people, } \\
\text { leave at }\end{array}$ & 3,103 & 484 & 494 & 12,133 & 1,972 & 1,976 \\
\hline Train & & & & 15,367 & 1,708 & 1,726 \\
\hline
\end{tabular}

Table 9: Statistics of the slot value vocabulary size in MultiWOZ 2.1.

\begin{tabular}{|c|c|c|c|}
\hline \multirow[b]{2}{*}{ Slot Name } & \multicolumn{3}{|c|}{ Slot Value Vocabulary Size } \\
\hline & Train & Valid & Test \\
\hline taxi-destination & 373 & 213 & 213 \\
\hline taxi-departure & 357 & 214 & 203 \\
\hline restaurant-name & 202 & 162 & 162 \\
\hline attraction-name & 186 & 145 & 149 \\
\hline train-leaveat & 146 & 69 & 117 \\
\hline train-arriveby & 112 & 64 & 101 \\
\hline restaurant-food & 111 & 81 & 70 \\
\hline taxi-leaveat & 105 & 68 & 65 \\
\hline hotel-name & 93 & 65 & 58 \\
\hline restaurant-book time & 64 & 50 & 51 \\
\hline taxi-arriveby & 95 & 49 & 46 \\
\hline train-destination & 27 & 25 & 24 \\
\hline train-departure & 34 & 23 & 23 \\
\hline attraction-type & 31 & 17 & 17 \\
\hline train-book people & 11 & 9 & 9 \\
\hline hotel-book people & 8 & 8 & 8 \\
\hline restaurant-book people & 9 & 8 & 8 \\
\hline hotel-book day & 13 & 7 & 7 \\
\hline hotel-stars & 9 & 7 & 7 \\
\hline restaurant-book day & 10 & 7 & 7 \\
\hline train-day & 8 & 7 & 7 \\
\hline attraction-area & 7 & 6 & 6 \\
\hline hotel-area & 7 & 6 & 6 \\
\hline restaurant-area & 7 & 6 & 6 \\
\hline hotel-book stay & 10 & 5 & 5 \\
\hline hotel-parking & 4 & 4 & 4 \\
\hline hotel-pricerange & 7 & 5 & 4 \\
\hline hotel-type & 5 & 5 & 4 \\
\hline restaurant-pricerange & 5 & 4 & 4 \\
\hline hotel-internet & 3 & 3 & 3 \\
\hline
\end{tabular}


Table 10: Statistics of domain transition in the test set of MultiWOZ 2.1. There are 140 dialogues with more than one domain transition that end with taxi domain. The cases where domain switches more than once and ends in taxi are shown in bold. The total number of dialogues with more than one domain transition is 175 . We can view these as complicated dialogues.

\begin{tabular}{|c|c|c|c|c|}
\hline \multicolumn{4}{|c|}{ Domain Transition } & \multirow[b]{2}{*}{ Count } \\
\hline First & Second & Third & Fourth & \\
\hline restaurant & train & - & - & 87 \\
\hline attraction & train & - & - & 80 \\
\hline hotel & - & - & - & 71 \\
\hline train & attraction & - & - & 71 \\
\hline train & hotel & - & - & 70 \\
\hline restaurant & - & - & - & 64 \\
\hline train & restaurant & - & - & 62 \\
\hline hotel & train & - & - & 57 \\
\hline $\operatorname{taxi}$ & - & - & - & 51 \\
\hline attraction & restaurant & - & - & 38 \\
\hline restaurant & attraction & taxi & - & 35 \\
\hline restaurant & attraction & - & - & 31 \\
\hline train & - & - & - & 31 \\
\hline hotel & attraction & - & - & 27 \\
\hline restaurant & hotel & - & - & 27 \\
\hline restaurant & hotel & $\operatorname{taxi}$ & - & 26 \\
\hline attraction & hotel & $\operatorname{taxi}$ & - & 24 \\
\hline attraction & restaurant & taxi & - & 23 \\
\hline hotel & restaurant & - & - & 22 \\
\hline attraction & hotel & - & - & 20 \\
\hline hotel & attraction & taxi & - & 16 \\
\hline hotel & restaurant & taxi & - & 13 \\
\hline attraction & - & - & - & 12 \\
\hline attraction & restaurant & train & - & 3 \\
\hline restaurant & hotel & train & - & 3 \\
\hline hotel & train & restaurant & - & 3 \\
\hline restaurant & train & hotel & - & 3 \\
\hline restaurant & $\operatorname{taxi}$ & hotel & - & 3 \\
\hline attraction & train & restaurant & - & 2 \\
\hline train & attraction & restaurant & - & 2 \\
\hline attraction & restaurant & hotel & - & 2 \\
\hline hotel & train & attraction & - & 2 \\
\hline attraction & $\operatorname{taxi}$ & hotel & - & 1 \\
\hline hotel & $\operatorname{taxi}$ & - & - & 1 \\
\hline train & hotel & restaurant & - & 1 \\
\hline restaurant & taxi & - & - & 1 \\
\hline restaurant & train & $\operatorname{taxi}$ & - & 1 \\
\hline hotel & restaurant & train & - & 1 \\
\hline hotel & $\operatorname{taxi}$ & train & - & 1 \\
\hline $\operatorname{taxi}$ & attraction & - & - & 1 \\
\hline restaurant & train & attraction & - & 1 \\
\hline attraction & train & hotel & - & 1 \\
\hline attraction & train & taxi & - & 1 \\
\hline restaurant & attraction & train & - & 1 \\
\hline hotel & taxi & attraction & - & 1 \\
\hline train & hotel & attraction & - & 1 \\
\hline restaurant & $\operatorname{taxi}$ & attraction & - & 1 \\
\hline hotel & attraction & restaurant & $\operatorname{taxi}$ & 1 \\
\hline attraction & hotel & train & - & 1 \\
\hline $\operatorname{taxi}$ & restaurant & train & - & 1 \\
\hline
\end{tabular}




\section{B Inference Time Complexity (ITC)}

Table 11: Inference Time Complexity (ITC) of each model. We report the ITC in both the best case and the worst case for more precise comparison. $J$ indicates the number of slots, and $M$ indicates the number of values of a slot.

\begin{tabular}{lll}
\hline & \multicolumn{2}{c}{ Inference Time Complexity } \\
\cline { 2 - 3 } Model & Best & Worst \\
\hline SUMBT & $\Omega(J M)$ & $O(J M)$ \\
DS-DST & $\Omega(J)$ & $O(J M)$ \\
DST-picklist & $\Omega(J M)$ & $O(J M)$ \\
DST Reader & $\Omega(1)$ & $O(J)$ \\
TRADE & $\Omega(J)$ & $O(J)$ \\
COMER & $\Omega(1)$ & $O(J)$ \\
NADST & $\Omega(1)$ & $O(1)$ \\
ML-BST & $\Omega(J)$ & $O(J)$ \\
SOM-DST(ours) & $\Omega(1)$ & $O(J)$ \\
\hline
\end{tabular}

Inference Time Complexity (ITC) proposed by Ren et al. (2019) defines the efficiency of a DST model using $J$, the number of slots, and $M$, the number of values of a slot. Going a step further from their work, we report ITC of the models in the best case and the worst case for relatively more precise comparison.

Table 11 shows ITC of several models in their best and worst cases. Since our model generates values for only the slots on which UPDATE operation has to be performed, the best case complexity of our model is $\Omega(1)$, when there is no slot whose operation is UPDATE. 


\section{Sample Outputs}

\begin{tabular}{|c|c|c|c|c|}
\hline Turn & Dialogue & $\begin{array}{c}\text { Pred } B_{t-1} \\
\text { (Model Output at Turn } t-1 \text { ) }\end{array}$ & $\begin{array}{c}\text { Pred } B_{t} \\
\text { (Model Output at Turn } t \text { ) }\end{array}$ & Ground Truth $B_{t}$ \\
\hline 1 & $\begin{array}{l}\text { U: i am looking for a cheap restaurant in the } \\
\text { center of the city. }\end{array}$ & & $\begin{array}{l}\text { restaurant-area-centre } \\
\text { restaurant-pricerange-cheap }\end{array}$ & $\begin{array}{l}\text { restaurant-area-centre } \\
\text { restaurant-pricerange-cheap }\end{array}$ \\
\hline 2 & $\begin{array}{l}\text { A: do you have any specific type of food you } \\
\text { would like? } \\
\text { U: no, i am not picky as long as the price -s are } \\
\text { low. }\end{array}$ & $\begin{array}{l}\text { restaurant-area-centre } \\
\text { restaurant-pricerange-cheap }\end{array}$ & $\begin{array}{l}\text { restaurant-area-centre } \\
\text { restaurant-pricerange-cheap } \\
\text { restaurant-food-dontcare }\end{array}$ & $\begin{array}{l}\text { restaurant-area-centre } \\
\text { restaurant-pricerange-cheap }\end{array}$ \\
\hline 3 & $\begin{array}{l}\text { A: there is a cheap chinese restaurant called the } \\
\text { dojo noodle bar located in the centre of town. } \\
\text { would you like to book a table? } \\
\text { U: yes please, for } 8 \text { people at } 18: 30 \text { on thursday. }\end{array}$ & $\begin{array}{l}\text { restaurant-area-centre } \\
\text { restaurant-pricerange-cheap } \\
\text { restaurant-food-dontcare }\end{array}$ & $\begin{array}{l}\text { restaurant-area-centre } \\
\text { restaurant-pricerange-cheap } \\
\text { restaurant-food-dontcare } \\
\text { restaurant-book time-18:30 } \\
\text { restaurant-book people-8 } \\
\text { restaurant-book day-thursday }\end{array}$ & $\begin{array}{l}\text { restaurant-area-centre } \\
\text { restaurant-pricerange-cheap } \\
\text { restaurant-book time-18:30 } \\
\text { restaurant-book people-8 } \\
\text { restaurant-book day-thursday } \\
\text { restaurant-name-dojo noodle bar }\end{array}$ \\
\hline 4 & $\begin{array}{l}\text { A: i am sorry but dojo noodle bar is solidly } \\
\text { booked at that time } \text { i can try a different time or } \\
\text { day for you. } \\
\text { U: can you try to book it at } 17: 30 \text {. }\end{array}$ & $\begin{array}{l}\text { restaurant-area-centre } \\
\text { restaurant-pricerange-cheap } \\
\text { restaurant-food-dontcare } \\
\text { restaurant-book time-18:30 } \\
\text { restaurant-book people-8 } \\
\text { restaurant-book day-thursday }\end{array}$ & $\begin{array}{l}\text { restaurant-area-centre } \\
\text { restaurant-pricerange-cheap } \\
\text { restaurant-food-dontcarc } \\
\text { restaurant-book time-17:30 } \\
\text { restaurant-book people-8 } \\
\text { restaurant-book day-thursday } \\
\text { restaurant-name-dojo noodle bar }\end{array}$ & $\begin{array}{l}\text { restaurant-area-centre } \\
\text { restaurant-pricerange-cheap } \\
\text { restaurant-book time-17:30 } \\
\text { restaurant-book people--8 } \\
\text { restaurant-book day-thursday } \\
\text { restaurant-name-dojo noodle bar }\end{array}$ \\
\hline 5 & $\begin{array}{l}\text { A: all set. your reference number is } \mathrm{k} 2 \mathrm{bo} 09 \mathrm{vq} \text {. } \\
\mathrm{U} \text { : thanks. } \mathrm{i} \text { am also looking for some } \\
\text { entertainment close to the restaurant. any } \\
\text { suggestions? }\end{array}$ & $\begin{array}{l}\text { restaurant-area-centre } \\
\text { restaurant-pricerange-cheap } \\
\text { restaurant-food-dontcare } \\
\text { restaurant-book time-17:30 } \\
\text { restaurant-book people-8 } \\
\text { restaurant-book day-thursday } \\
\text { restaurant-name-dojo noodle bar }\end{array}$ & $\begin{array}{l}\text { restaurant-area-centre } \\
\text { restaurant-pricerange-cheap } \\
\text { restaurant-food-dontcarc } \\
\text { restaurant-book time-17:30 } \\
\text { restaurant-book people-8 } \\
\text { restaurant-book day-thursday } \\
\text { restaurant-name-dojo noodle bar } \\
\text { attraction-area-centre } \\
\text { attraction-type-entertainment }\end{array}$ & $\begin{array}{l}\text { restaurant-area-centre } \\
\text { restaurant-pricerange-cheap } \\
\text { restaurant-book time-17:30 } \\
\text { restaurant-book people-8 } \\
\text { restaurant-book day-thursday } \\
\text { restaurant-name-dojo noodle bar } \\
\text { attraction-area-centre } \\
\text { attraction-type-entertainment }\end{array}$ \\
\hline 6 & $\begin{array}{l}\text { A: } i \text { am sorry I do not have any attractions } \\
\text { meeting the criteria you listed. is there another } \\
\text { type of attraction or area you would like me to } \\
\text { search? } \\
\text { U: why do not you try an architectural attraction, } \\
\text { instead. }\end{array}$ & $\begin{array}{l}\text { restaurant-arca-centre } \\
\text { restaurant-pricerange-cheap } \\
\text { restaurant-food-dontcare } \\
\text { restaurant-book time-17:30 } \\
\text { restaurant-book people-8 } \\
\text { restaurant-book day-thursday } \\
\text { restuarant-name-djo noodle bar } \\
\text { attraction-area-centre } \\
\text { attraction-type-entertainment }\end{array}$ & $\begin{array}{l}\text { restaurant-area-centre } \\
\text { restaurant-pricerange-cheap } \\
\text { restaurant-food-dontcare } \\
\text { restaurant-book time-17:30 } \\
\text { restaurant--book people-8 } \\
\text { restaurant-book day-thursday } \\
\text { restaurant-name-dojo noodle bar } \\
\text { attraction-area-centre } \\
\text { attraction-type-architecture }\end{array}$ & $\begin{array}{l}\text { restaurant-area-centre } \\
\text { restaurant-pricerange-cheap } \\
\text { restaurant-book time-17:30 } \\
\text { restaurant-book people-8 } \\
\text { restaurant-book day-thursday } \\
\text { Rrstaurant-name-dojo noodle bar } \\
\text { attraction-area-centre } \\
\text { attraction-type-architecture }\end{array}$ \\
\hline 7 & $\begin{array}{l}\text { A: all saints church looks good, would you like } \\
\text { to head there? } \\
\text { U: yes please, can you let me know what the } \\
\text { entrance fee is too? }\end{array}$ & $\begin{array}{l}\text { restaurant-area-centre } \\
\text { restaurant-pricerange-cheap } \\
\text { restaurant-food-dontcare } \\
\text { restaurant-book time-17:30 } \\
\text { restaurant-book people-8 } \\
\text { restaurant-book day-thursday } \\
\text { restaurant-name-dojo noodle bar } \\
\text { attraction-area-centre } \\
\text { attraction-type-architecture }\end{array}$ & 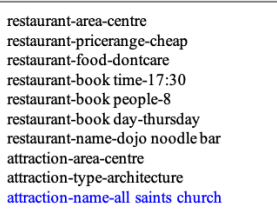 & $\begin{array}{l}\text { restaurant-area-centre } \\
\text { restaurant-pricerange-cheap } \\
\text { restaurant-book time-17:30 } \\
\text { restaurant-book people-8 } \\
\text { restaurant-book day-thursday } \\
\text { restaurant-name-dojo noodle bar } \\
\text { attraction-area-centre } \\
\text { attraction-type-architecture } \\
\text { attraction-name-all saints church }\end{array}$ \\
\hline 8 & $\begin{array}{l}\text { A: its entrance fee is free . } \\
\mathrm{U}: \mathrm{i} \text { also need to book a taxi between the } \\
\text { restaurant and the church. }\end{array}$ & $\begin{array}{l}\text { restaurant-area-centre } \\
\text { restaurant-pricerange-cheap } \\
\text { restaurant-food-dontcare } \\
\text { restaurant-book time-17:30 } \\
\text { restaurant-book people-8 } \\
\text { restaurant-book day-thursday } \\
\text { restaurant-name-dojo noodle bar } \\
\text { attraction-area-centre } \\
\text { attraction-type-architecture } \\
\text { attraction-name-all saints church }\end{array}$ & $\begin{array}{l}\text { restaurant-area-centre } \\
\text { restaurant-pricerange-cheap } \\
\text { restaurant-food-dontcare } \\
\text { restaurant-book time-17: } 30 \\
\text { restaurant-book people-8 } \\
\text { restaurant-book day-thursday } \\
\text { restaurant-name-dojo noodle bar } \\
\text { attraction-area-centre } \\
\text { attraction-type-architecture } \\
\text { attraction-name-all saints church } \\
\text { taxi-departure-dojo noodle bar }\end{array}$ & $\begin{array}{l}\text { restaurant-area-centre } \\
\text { restaurant-pricerange-cheap } \\
\text { restaurant-book time-17:30 } \\
\text { restaurant-book people-8 } \\
\text { restaurant-book day-thursday } \\
\text { restaurant-name-dojo noodle bar } \\
\text { attraction-area-centre } \\
\text { attraction-type-architecture } \\
\text { attraction-name-all saints church } \\
\text { taxi-departure-dojo noodle bar } \\
\text { taxi-destination-all saints church }\end{array}$ \\
\hline 9 & $\begin{array}{l}\text { A: what time would you like the taxi to pick you } \\
\text { up from dojo noodle bar? } \\
\text { U: } 18: 30 \text {, please. }\end{array}$ & $\begin{array}{l}\text { restaurant-area-centre } \\
\text { restaurant-pricerange-cheap } \\
\text { restaurant-food-dontcare } \\
\text { restaurant-book time-17:30 } \\
\text { restaurant-book people-8 } \\
\text { restaurant-book day-thursday } \\
\text { restaurant-name-dojo noodle bar } \\
\text { attraction-area-centre } \\
\text { attraction-type-architecture } \\
\text { attraction-name-all saints church } \\
\text { taxi-departure-dojo noodle bar }\end{array}$ & $\begin{array}{l}\text { restaurant-area-centre } \\
\text { restaurant-pricerange-cheap } \\
\text { restaurant-food-dontcare } \\
\text { restaurant-book time-17:30 } \\
\text { restaurant-book people-8 } \\
\text { restaurant-book day-thursday } \\
\text { restaurant-name-dojo noodle bar } \\
\text { attraction-area-centre } \\
\text { attraction-type-architecture } \\
\text { attraction-name-all saints church } \\
\text { taxi-departure-dojo noodleb bar } \\
\text { taxi-leaveat-18:30 }\end{array}$ & $\begin{array}{l}\text { restaurant-area-centre } \\
\text { restaurant-pricerange-cheap } \\
\text { restaurant-book time-17:30 } \\
\text { restaurant-book people-8 } \\
\text { restaurant-book day-thursday } \\
\text { restaurant-name-dojo noodle bar } \\
\text { attraction-area-centre } \\
\text { attraction-type-architecture } \\
\text { attraction-name-all saints church } \\
\text { taix-departure-dojo noodle bar } \\
\text { taxi-destination-all saints church } \\
\text { taxi-leaveat-18:30 }\end{array}$ \\
\hline 10 & $\begin{array}{l}\text { A: your taxi is booked. the car is a blue bmw } \\
\text { and the contact number is } 07023944669 . \text { is there } \\
\text { anything else I can help you with? } \\
\text { U: that s it . thanks. }\end{array}$ & $\begin{array}{l}\text { restaurant-area-centre } \\
\text { restaurant-pricerange-cheap } \\
\text { restaurant-food-dontcare } \\
\text { restaurant-book time-17:30 } \\
\text { restaurant-book people-8 } \\
\text { restaurant-book day-thursday } \\
\text { restaurant-name-dojo noodle bar } \\
\text { attraction-area-centre } \\
\text { attraction-type-architecture } \\
\text { attraction-name-all saints church } \\
\text { taxi-departure-dojo noodle bar } \\
\text { taxi-leaveat-18:30 }\end{array}$ & $\begin{array}{l}\text { restaurant-area-centre } \\
\text { restaurant-pricerange-cheap } \\
\text { restaurant-food-dontcare } \\
\text { restaurant-book time-17:30 } \\
\text { restaurant-took people-8 } \\
\text { restaurant-book day-thursday } \\
\text { restaurant-name-dojo noodlebar } \\
\text { attraction-area-centre } \\
\text { attraction-type-architecture } \\
\text { attraction-name-all saints church } \\
\text { taxi-departure-dojo noodlebar } \\
\text { taxi-leaveat-1 } 18: 30\end{array}$ & $\begin{array}{l}\text { restaurant-area-centre } \\
\text { restaurant-pricerange-cheap } \\
\text { restaurant-book time-17:30 } \\
\text { restaurant-book people-8 } \\
\text { restaurant-book day-thursday } \\
\text { restaurant-name-dojo noodle bar } \\
\text { attraction-area-centre } \\
\text { attraction-type-architecture } \\
\text { attraction-name-all saints church } \\
\text { taxi-departure-dojo noodle bar } \\
\text { taxi-destination-all saints church } \\
\text { taxi-leaveat-18:30 }\end{array}$ \\
\hline
\end{tabular}

Figure 3: The output of SOM-DST in a dialogue (dialogue_idx MUL2499) in the test set of MultiWOZ 2.1. Parts changed from the previous dialogue state are shown in blue. To save space, we omit the slots with value NULL from the figure. 


\begin{tabular}{|c|c|c|c|c|}
\hline Turn & Dialogue & $\begin{array}{c}\text { Pred } B_{t-1} \\
\text { (Model Output at Turn } t-1)\end{array}$ & $\begin{array}{c}\text { Pred } B_{t} \\
\text { (Model Output at Turn } t \text { ) }\end{array}$ & Ground Truth $B_{t}$ \\
\hline 1 & $\begin{array}{l}\mathrm{U}: \mathrm{i} \text { am planing a trip to cambridge and i need } \\
\text { ideas on a place } \mathrm{i} \text { can go in the centre }\end{array}$ & & attraction-area-centre & attraction-area-centre \\
\hline 2 & $\begin{array}{l}\text { A: there is much to choose from in the centre. } \\
\text { there are several architecture attractions to } \\
\text { explore, boats and swimming, colleges, } \\
\text { museums, parks, theatres and even nightclubs. } \\
\text { U: what do you recommend? }\end{array}$ & attraction-area-centre & attraction-area-centre & attraction-area-centre \\
\hline 3 & $\begin{array}{l}\text { A: i recommend whipple museum of the history } \\
\text { of science. it s educational and free ! } \\
\text { U: okay that sounds fun. }\end{array}$ & attraction-area-centre & $\begin{array}{l}\text { attraction-area-centre } \\
\text { attraction-name-whipple museum of the } \\
\text { history of science }\end{array}$ & $\begin{array}{l}\text { attraction-area-centre } \\
\text { attraction-name-whipple museum of the } \\
\text { history of science }\end{array}$ \\
\hline 4 & $\begin{array}{l}\text { A: is there anything else } \mathrm{i} \text { can help you with } \\
\text { today? } \\
\text { U: what can you tell me about the express by } \\
\text { holiday inn cambridge? }\end{array}$ & $\begin{array}{l}\text { attraction-area-centre } \\
\text { attraction-name-whipple museum of the } \\
\text { history of science }\end{array}$ & $\begin{array}{l}\text { attraction-area-centre } \\
\text { attraction-name-whipple museum of the } \\
\text { history of science } \\
\text { hotel-name-express by holiday inn } \\
\text { cambridge }\end{array}$ & $\begin{array}{l}\text { attraction-area-centre } \\
\text { attraction-name-whipple museum of the } \\
\text { history of science } \\
\text { hotel-name-express by holiday inn } \\
\text { cambridge }\end{array}$ \\
\hline 5 & $\begin{array}{l}\text { A: sure it is located in the east and is in the } \\
\text { expensive price range and their telephone } \\
\text { number is } 01223866800 \text {. } \\
\text { U: can you book it for me please ? i need it for } 1 \\
\text { person and } 5 \text { nights starting on tuesday. }\end{array}$ & $\begin{array}{l}\text { attraction-area-centre } \\
\text { attraction-name-whipple museum of the } \\
\text { history of science } \\
\text { hotel-name-express by holiday inn } \\
\text { cambridge }\end{array}$ & $\begin{array}{l}\text { attraction-area-centre } \\
\text { attraction-name-whipple museum of the } \\
\text { history of science } \\
\text { hotel-name-express by holiday inn } \\
\text { cambridge } \\
\text { hotel-book day-5 } \\
\text { hotel-book people-1 } \\
\text { hotel-book day-thuesday }\end{array}$ & $\begin{array}{l}\text { attraction-arca-centre } \\
\text { attraction-name-whipple museum of the } \\
\text { history of coience } \\
\text { hotel-name-express by holiday inn } \\
\text { canbridge } \\
\text { hotel-book day-5 } \\
\text { hotel-book people-1 } \\
\text { hotel-book day-thuesday }\end{array}$ \\
\hline 6 & $\begin{array}{l}\text { A: i was able to book your stay at the express by } \\
\text { holiday inn cambridge . your reference number } \\
\text { is ye0m6sg } 1 \text {. } \\
\text { U: great ! thanks. i was also hoping to book a } \\
\text { taxi, can you help me with that? }\end{array}$ & $\begin{array}{l}\text { attraction-area-centre } \\
\text { attraction-name-whipple museum of the } \\
\text { history of science } \\
\text { hotel-name-express by holiday inn } \\
\text { cambridge } \\
\text { hotel-book day-5 } \\
\text { hotel-book people-1 } \\
\text { hotel-book day-thuesday }\end{array}$ & $\begin{array}{l}\text { attraction-area-centre } \\
\text { attraction-name-whipple museum of the } \\
\text { history of science } \\
\text { hotel-name-express by holiday inn } \\
\text { cambridge } \\
\text { hotel-book day-5 } \\
\text { hotel-book people-1 } \\
\text { hotel-book day-thuesday }\end{array}$ & $\begin{array}{l}\text { attraction-area-centre } \\
\text { attraction-name-whipple museum of the } \\
\text { history of sciencec } \\
\text { hotel-name-express by holiday inn } \\
\text { canbridge } \\
\text { hotel-book day- } 5 \\
\text { hotel-book people-1 } \\
\text { hotel-book day-thuesday }\end{array}$ \\
\hline 7 & $\begin{array}{l}\text { A: yes } i \text { can, what is the departure and } \\
\text { destionation site, and when would you like to } \\
\text { leave and arrive by? } \\
\text { U: i would like to leave the hotel by } 02: 45 \text { to go } \\
\text { to the museum. }\end{array}$ & $\begin{array}{l}\text { attraction-area-centre } \\
\text { attraction-name-whipple museum of the } \\
\text { history of fcience } \\
\text { hotel-name-express by holiday inn } \\
\text { cambridge } \\
\text { hotel-book day-5 } \\
\text { hotel-book people-1 } \\
\text { hotel-book day-thuesday }\end{array}$ & $\begin{array}{l}\text { attraction-area-centre } \\
\text { attraction-name-whipple museum of the } \\
\text { history of science } \\
\text { hotel-namc-express by holiday inn } \\
\text { cambridge } \\
\text { hotel-book day-5 } \\
\text { hotel-book people-1 } \\
\text { hotel-book day-thuesday } \\
\text { taxi-departure-express by holiday inn } \\
\text { cambridge } \\
\text { taxi-destination-whipple-museum of the } \\
\text { history of science } \\
\text { taxi-leaveat-02:45 }\end{array}$ & $\begin{array}{l}\text { attraction-area-centre } \\
\text { attraction-name-whipple museum of the } \\
\text { history of fciencec } \\
\text { hotel-name-express by holiday inn } \\
\text { canbridge } \\
\text { hotel-book day- } 5 \\
\text { hotel-book people-1 } \\
\text { hotel-book day-thuesday } \\
\text { taxi-departure-express by holiday inn } \\
\text { cambridge } \\
\text { taxi-destination-whipple-museum of the } \\
\text { history of science } \\
\text { taxi-leaveat--02:45 }\end{array}$ \\
\hline 8 & $\begin{array}{l}\text { A: okay, } i \text { have got that booked for you . you are } \\
\text { taxi is a yellow honda and the contact number is } \\
07272096370 . \text { can i do anything else today? } \\
\text { U: thanks alot for helping }\end{array}$ & $\begin{array}{l}\text { attraction-area-centre } \\
\text { attraction-name-whipple museum of the } \\
\text { history of science } \\
\text { hotel-name-express by holiday inn } \\
\text { cambridge } \\
\text { hotel-book day-5 } \\
\text { hotel-book people-1 } \\
\text { hotel-book day-thuesday } \\
\text { taxi-departure-express by holiday inn } \\
\text { cambridge } \\
\text { taxi-destination-whipple-museum of the } \\
\text { history of science } \\
\text { taxi-leaveat-02:45 }\end{array}$ & $\begin{array}{l}\text { attraction-area-centre } \\
\text { attraction-name-whipple museum of the } \\
\text { history of science } \\
\text { hotel-name-express by holiday inn } \\
\text { cambridge } \\
\text { hotel-1ook day-5 } \\
\text { hotel-book people-1 } \\
\text { hotel-book day-thuesday } \\
\text { taxi-departure-express by holiday inn } \\
\text { cambridge } \\
\text { taxi-destination-whipple-museum of the } \\
\text { history of science } \\
\text { taxi-leaveat-02:45 }\end{array}$ & $\begin{array}{l}\text { attraction-arca-centre } \\
\text { attraction-name-whipple museum of the } \\
\text { history of science } \\
\text { hotel-name-express by holiday inn } \\
\text { canbridge } \\
\text { hotel-book day- } 5 \\
\text { hotel-book people-1 } \\
\text { hotel-book day-thuesday } \\
\text { taxi-departure-express by holiday inn } \\
\text { cambridge } \\
\text { taxi-destination-whipple-museum of the } \\
\text { history of colience } \\
\text { taxi-leaveat-02:45 }\end{array}$ \\
\hline
\end{tabular}

Figure 4: The output of SOM-DST in a dialogue (dialogue_idx PMUL3748) in the test set of MultiWOZ 2.1. Parts changed from the previous dialogue state are shown in blue. To save space, we omit the slots with value NULL from the figure. 